\title{
Crossable Surfaces of Robotic Manipulators with Joint Limits
}

\author{
Karim Abdel-Malek \\ Harn-Jou Yeh \\ Department of Mechanical Engineering \\ The University of Iowa \\ Iowa City, IA 52242 \\ Tel. (319) 335-5676 Fax. (319) 335-5669 \\ amalek@engineering.uiowa.edu
}

\begin{abstract}
A broadly applicable formulation for determining the crossability of singular surfaces and curves in the workspace of serial robotic manipulators is presented. Singular surfaces and curves are analytically determined using nullspace rank-deficiency criteria of the mechanism's constraint position Jacobian. Imposed joint limits in terms of inequality constraints are taken into account in the formulation. Directions of admissible normal movements on a surface or curve are established from the analysis of the normal curvature of singular surfaces. The normal curvature of a parametric surface used in this formulation, is determined from the first and second fundamental forms adapted from differential geometry. Definiteness properties of a quadratic form developed from the acceleration analysis determine admissible normal movements. For singular surfaces resulting from active joint constraints, definiteness properties are not enough and supplementary criteria are necessary. Two additional criteria are derived. This paper is a complete treatment of the problem of determining whether a singular surface/curve in the workspace is crossable. Planar and spatial numerical examples are presented to illustrate the formulation.
\end{abstract}

\section{Introduction}

The problem of determining singular behavior of mechanical manipulators has received considerable attention. The reciprocal screw method for workspace generation, for example, is based on the fact that when the hand reference point of the manipulator is on the workspace boundary when the manipulator has a spherical wrist, all the joint axes of a manipulator are reciprocal to a zero pitch wrench axis (Sugimoto 1982). For each degree-of-freedom (DOF) lost, there exists one reciprocal screw that, if applied as a wrench to the end-effector, produces no virtual work for the manipulator joints. Wang and Waldron (1986), based upon earlier work (Waldron, et al. 1985), stated that as the Jacobian of the manipulator becomes singular, its columns, which are screw quantities, do not span the full rank of the matrix, therefore, the Jacobian rank decreases by at least one. Proof of the nullspace criteria applied to the Jacobian was presented by Spanos and Kohli (1985).

Other methods that are based on Jacobian singularity can be found in (Shu, et al. 1983, Litvin, et al. 1986a, and 1986b). An enumeration of singular configurations due to the vanishing of the determinant of the Jacobian and the Jacobian's minors is presented by Lipkin and Pohl (1991). Shamir (1990) provided an analytical tool to determine if the singularities are avoidable for three 
DOF manipulators. Gorla (1981) was able to get expressions for the set of singular points by assuming that link twists were multiple of $\pi / 2$. Geometric approaches to the study of singular configurations of a manipulator arm were addressed by Lai and Yang (1986), Ahmad and Luo (1988), and Tourassis and Ang (1992). Other earlier important studies that discussed manipulator singularities include Soylu and Duffy (1988) and Lai and Yang (1984).

Early studies that have addressed difficulties in the control of manipulators due to the appearance of interior curves and surfaces were reported by Waldron (1987) and Nielsen, et al. (1991). In the latter work, the controllability of a mechanical arm is discussed from a differential geometry point of view. Nielsen, et al. showed control difficulties based upon vector fields and their consecutive Lie brackets spanning a state space. A criterion to define possible motion (socalled feasible trajectory) from a singularity was presented by Chevallereau and Daya (1994) and Chevallereau (1996).

The fundamental concept of crossable and noncrossable surfaces inside a manipulator's workspace was addressed by Oblak and Kohli (1988). Although their report has touched upon a crossable surface using the singularity criteria of the Jacobian, the paper does not present a unified method for identifying a crossable surface or curve, neither does it determine directions of admissible movement. Further work on the issue of singularity determination and the structure of singular regions inside the workspace was presented by Pai and Leu (1992), Burdick (1991; 1992), Karger (1995), and Merlet (1989).

Recently, Haug, et al. (1995; 1996) presented a numerical algorithm for identifying and analyzing barriers to output control of manipulators using first and second-order Taylor approximations of the output in selected directions. Haug, et al. showed that the output velocity in the direction normal to such curves and surfaces must be zero. The work was extended to the investigation of the domains of mobility for a planar body moving among obstacles (Haug, et al. 1998) and to the study the workspace (called operational envelope) of the Stewart platform (Adkins and Haug 1997). It should be noted that this method is completely numerical, yields only curves on the boundary that must be manually meshed to depict the envelope. Similar work based on the Euler-Rodrigues parameters and aimed at determining the workspace of serial and platform manipulators through the occurrence of singular behavior (Bulca, et al. 1999).

Recently, Abdel-Malek and Yeh (1997a) presented a general method by which all singular surfaces and curves can be analytically determined for a 3DOF serial manipulator and hence obtained a closed form of the surface patches enveloping the workspace. The method and algorithm were extended to the concept of swept volumes whereby the volume generated by the motion of a geometric entity in space is numerically determined (Abdel-Malek and Yeh 1997b) and was shown to apply to solid modeling. The concept of singular curves and surfaces was then used to determine feasible path trajectories where the end-effector can cross a given barrier (Abdel-Malek and Yeh 1997c). The formulation therein was compared with the results of the numerical method by Haug, et al. (1996) in a joint paper (Abdel-Malek, et al. 1997). The same theory implemented for the determination of the serial 5-DOF manipulator was presented by 
Abdel-Malek, et al. (1999) and reported singular surfaces that are boundary to the workspace but that can be further stratified.

We first present a rigorous formulation for identifying singular curves and surfaces internal and on the boundary to the workspace envelope. We then adapt methods from differential geometry to investigate properties associated with a proposed acceleration function on these curves and surfaces. A number of examples are presented to demonstrate the theory and formulation.

\section{Formulation}

It has been shown (Abdel-Malek and Yeh 1997a) that singular surfaces/curves comprising the workspace of serial robotic manipulators, can analytically be determined by studying the analytic Jacobian of the mechanism. For a position vector $\mathbf{x}=\boldsymbol{\Phi}(\mathbf{q})$, where $\mathbf{q} \in \mathbf{R}^{n}$ is the vector of generalized coordinates of an $n$-DOF manipulator, the dimension of the nullspace of the Jacobian $\boldsymbol{\Phi}_{\mathbf{q}}=\left[\partial \Phi_{i} / \partial q_{j}\right]$ is associated with singular behavior. Define the subvector of $\mathbf{q}$ as $\mathbf{p}_{i}=\mathbf{R}^{m}$, where $m \leq n-1$ such that $\mathbf{q} \equiv \mathbf{u} \cup \mathbf{p}_{i}$, where $\mathbf{u} \in \mathbf{R}^{n-m}$.

Generalized coordinates called singular sets, are determined from

$$
\mathbf{S}=\left\{\mathbf{p}_{i} ; \operatorname{dim}\left[\operatorname{Null}\left(\boldsymbol{\Phi}_{\mathbf{q}}^{T}(\mathbf{q})\right)\right] \geq 1, \mathbf{q}=\left[\begin{array}{ll}
\mathbf{u} & \mathbf{p}_{i}
\end{array}\right] \text {, for some } \mathbf{p}_{i}\right\}
$$

where $\mathbf{p}_{i}$ are joint variables that are either constant values of joints or defined as functions of other variables. The set of analytic functions generated by a subset of a Euclidean space defined by zeros of a finite number of differentiable functions (sub-determinants) are called varieties (singular sets). The nullspace of the Jacobian corresponds to joint velocities that do not produce any end-effector velocity for a given manipulator configuration $\mathbf{p}_{i}$. Singular configurations $\mathbf{p}_{i}$ are obtained from $\mathbf{S}$ and from the reduced-order manipulator condition discussed in AbdelMalek, et al. (1999), which occurs when joint constraints are active. It was shown that substituting $\mathbf{p}_{i}$ into $\boldsymbol{\Phi ( q )}$ yields singular surfaces and curves parameterized by $\Psi(\mathbf{u})$ where $\mathbf{u}$ is the new vector of generalized coordinates containing the remaining variables such that

$$
\Phi\left(\mathbf{u}, \mathbf{p}_{i}\right)=\Psi(\mathbf{u})
$$

A proof was presented showing that the basis of the null space of $\boldsymbol{\Phi}_{\mathbf{q}}^{T}$ is a normal vector to singular surfaces/curves in the workspace. i.e., if a vector $\mathbf{n}_{o}$ evaluated at $\mathbf{q}_{o}$ satisfies

$$
\boldsymbol{\Phi}_{\mathbf{q}}^{T} \mathbf{n}_{\mathrm{o}}=\mathbf{0}
$$

where $\mathbf{n}_{\mathrm{o}}=\left[\begin{array}{lll}\gamma_{1} & \gamma_{2} & \gamma_{3}\end{array}\right]^{T}$, it is the vector normal to singular surfaces/curves at $\mathbf{q}_{o}$. This important result was used in the velocity analysis of the end-effector on singular surfaces/curves to show that the end-effector on a singular surface may have only tangential velocities. Acceleration analysis of the end-effector revealed a quadratic form that can be used to establish possible directions of admissible movements. The normal acceleration is derived as

$$
a_{n}=\mathbf{n}_{o}^{T} \ddot{\mathbf{x}}=\dot{\mathbf{q}}^{T}\left[\mathbf{n}_{o}^{T} \boldsymbol{\Phi}\right]_{\mathbf{q q}} \dot{\mathbf{q}}
$$

where $\ddot{\mathbf{x}}$ is the acceleration of the end-effector, $\dot{\mathbf{q}}$ is the vector of joint velocities and two subscripts indicate a second derivative of $\left[\mathbf{n}_{o}^{T} \boldsymbol{\Phi}\right]$. Definiteness properties of the quadratic form 
in Eq. (4) predict the direction of admissible normal acceleration, but do not guarantee whether the end-effector can admit movement across a singular surface/curve (crossability analysis).

Consider the end-effector at a point on a singular surface with radius of curvature $\rho_{o}$, with a specified normal acceleration $a_{n}$, and a tangential velocity $v_{t}$. This manipulator will admit motion in one normal direction or another subject to

$$
a_{n}-\frac{\left|v_{t}^{2}\right|}{\rho_{o}}
$$

where $v_{t}=\dot{\mathbf{x}}=\Phi_{\mathbf{q}} \dot{\mathbf{q}}$ is the tangential velocity and $1 / \rho_{o}$ is the normal curvature of the singular surface with respect to the tangent direction of $v_{t}$ ( $\rho_{o}$ is the radius of curvature). The sign of (5) above establishes the admissible direction of motion.

From the theory of differential geometry (Farin 1993), the First and Second Fundamental Forms (denoted by $\mathbf{I}_{p}$ and $\mathbf{I}_{p}$, respectively) of a parametric geometric entity $\Psi(\mathbf{u})$, where $\mathbf{u}=\left[\begin{array}{ll}u & v\end{array}\right]^{T}$, is defined as

$$
\mathbf{I}_{p} \equiv \delta \mathbf{u}^{T} \Psi_{\mathbf{u}}^{T} \Psi_{\mathbf{u}} \delta \mathbf{u}
$$

where $\Psi_{\mathbf{u}}=\partial \Psi / \partial \mathbf{u}$ and $\Psi_{\mathbf{v}}=\partial \Psi / \partial \mathbf{v}$, and

$$
\mathbf{I I}_{p} \equiv \delta \mathbf{u}^{T}\left[\mathbf{n}^{T} \Psi\right]_{\mathbf{u u}} \delta \mathbf{u}
$$

or expanded as

$$
\mathbf{I I}_{p}=\mathbf{n}^{T} \boldsymbol{\Psi}_{u u} d u^{2}+2 \mathbf{n}^{T} \boldsymbol{\Psi}_{u v} d u d v+\mathbf{n}^{T} \boldsymbol{\Psi}_{v v} d v^{2}
$$

where $\mathbf{n}$ is the normal vector to the singular surface and $\Psi_{u v}=\partial^{2} \Psi / \partial u \partial v, \Psi_{v v}=\partial^{2} \Psi / \partial v \partial v$, and $\Psi_{u u}=\partial^{2} \Psi / \partial u \partial u$. The Normal Curvature $K_{o}$ of a parametric singular surface at a configuration $\mathbf{q}_{o}$, can then be defined as the ratio

$$
K_{o}=\frac{1}{\rho_{o}}=\frac{\mathbf{I I}_{p}}{\mathbf{I}_{p}}
$$

Define the time-Modified First and Second Fundamental Forms as

$$
\begin{gathered}
\mathbf{I}_{p}^{\prime} \equiv \dot{\mathbf{u}}^{T} \boldsymbol{\Psi}_{\mathbf{u}}^{T} \boldsymbol{\Psi}_{\mathbf{u}} \dot{\mathbf{u}} \\
\mathbf{I I}_{p}^{\prime} \equiv \dot{\mathbf{u}}^{T}\left[\mathbf{n}^{T} \boldsymbol{\Psi}\right]_{\mathbf{u u}} \dot{\mathbf{u}}
\end{gathered}
$$

such that the normal curvature can still be defined as

$$
K_{o}=\frac{1}{\rho_{o}}=\frac{\mathbf{I I}_{p}}{\mathbf{I}_{p}}=\frac{\mathbf{I I}_{p}^{\prime}}{\mathbf{I}_{p}^{\prime}}
$$

The tangent velocity in terms of $\Psi$ or $\Phi$ can be written as

$$
\Psi_{\mathbf{u}} \dot{\mathbf{u}}=\Phi_{\mathbf{q}} \dot{\mathbf{q}}
$$

Hence, it can be shown that the time-Modified First Fundamental Form is indeed the square of the tangential velocity

$$
\mathbf{I}_{p}^{\prime}=\left|v_{t}\right|^{2}
$$

Therefore, the difference in acceleration components can be written as 


$$
\begin{gathered}
a_{n}-\frac{\left|v_{t}\right|^{2}}{\rho_{o}}=a_{n}-\left|v_{t}\right|^{2} \frac{\mathbf{I I}_{p}^{\prime}}{\mathbf{I}_{p}^{\prime}} \\
=a_{n}-\mathbf{I I}_{p}^{\prime}
\end{gathered}
$$

Since $a_{n}$ is in terms of $\dot{\mathbf{q}}$ and to express $\mathbf{I I}_{p}^{\prime}$ in terms of $\dot{\mathbf{q}}$, it was shown that the velocity vector on a singular surface can be written as

$$
\dot{\mathbf{u}}=\left[\mathbf{E} \Psi_{\mathbf{u}}\right]^{-1} \mathbf{E} \Phi_{\mathbf{q}} \dot{\mathbf{q}}
$$

where

$$
\begin{aligned}
& \mathbf{E}=\left[\begin{array}{lll}
1 & 0 & 0 \\
0 & 1 & 0
\end{array}\right] \text { if the first and second rows of } \Psi_{\mathbf{u}} \text { are independent } \\
& \mathbf{E}=\left[\begin{array}{lll}
1 & 0 & 0 \\
0 & 0 & 1
\end{array}\right] \text { if the first and third rows of } \Psi_{\mathbf{u}} \text { are independent } \\
& \mathbf{E}=\left[\begin{array}{lll}
0 & 1 & 0 \\
0 & 0 & 1
\end{array}\right] \text { if the second and third rows of } \Psi_{\mathbf{u}} \text { are independent }
\end{aligned}
$$

For an end-effector on a singular surface, the crossability criteria was then expanded into a quadratic form written as

$$
a_{n}-\frac{\left|v_{t}\right|^{2}}{\rho_{o}}=a_{n}-\mathbf{I}_{p}^{\prime}=\dot{\mathbf{q}}^{T} \mathbf{Q} \dot{\mathbf{q}}
$$

where

$$
\mathbf{Q}=\left[\mathbf{n}^{T} \boldsymbol{\Phi}\right]_{\mathbf{q q}}-\boldsymbol{\Phi}_{\mathbf{q}}^{T} \mathbf{B}^{T}\left[\mathbf{n}^{T} \boldsymbol{\Psi}\right]_{\mathbf{u u}} \mathbf{B} \boldsymbol{\Phi}_{\mathbf{q}}
$$

and $\mathbf{B}$ is a generalized inverse of $\Psi_{\mathbf{u}}$ defined in Eq. (15) by

$$
\mathbf{B} \equiv\left[\mathbf{E} \Psi_{\mathbf{u}}\right]^{-1} \mathbf{E}
$$

Definiteness properties of the quadratic form in Eq. (17) indicate the crossability of a singular surface/curve. Since singular surfaces/curves that are non-crossable are impediments to motion, controllability issues are directly related to this formulation.

In this paper, we extend the formulation to manipulators with joint limits. The formulation includes joint parameters that are subject to mobility constraints. Since additional singular surfaces/curves may appear in the workspace due to active joint constraints, velocities of the joints that reached their limits on those surfaces are nulled. The nullification of joint velocities will give rise to an apparent loss of information from the matrix of the quadratic form (Eq. 17). Therefore, additional (supplementary) criteria are developed to determine admissible normal movements on those surfaces/curves.

\section{Extending the Formulation to Include Joint Limits}

Joint limits imposed in the form of inequality constraints such as $q_{i}^{\min } \leq q_{i} \leq q_{i}^{\max }$, are parameterized into an equality using the following transformation such that new generalized coordinates $\lambda_{i}$ are introduced

$$
q_{i}=a_{i}+b_{i} \sin \lambda_{i}
$$


where $a_{i}=\left(q_{i}{ }^{\max }+q_{i}{ }^{\min }\right) / 2$ and $b_{i}=\left(q_{i}{ }^{\max }-q_{i}{ }^{\min }\right) / 2$ are the mid point and half range of the inequality constraint (Haug, et al. 1994). The constraint function is then written in terms of the extended vector $\mathbf{s}=\left[\lambda_{1} \lambda_{2} \ldots \lambda_{n}\right]^{T}$ such that

$$
\mathbf{x}=\boldsymbol{\Phi}(\mathbf{s})=\left[\boldsymbol{\Phi}\left(\mathbf{q}\left(\lambda_{i}\right)\right)\right]
$$

Differentiating with respect to time and using the chain rule, the velocity of the end-effector is

$$
\dot{\mathbf{x}}=\Phi_{\mathrm{q}} \mathbf{q}_{\mathbf{s}} \dot{\mathbf{s}}
$$

where $\mathbf{q}_{\mathbf{s}}=\partial q_{i} / \partial \lambda_{j}$ and $\dot{\mathbf{s}}=d \mathbf{s} / d t$. On a singular surface, the term $\left.\boldsymbol{\Phi}_{\mathbf{q}} \mathbf{q}_{\mathbf{s}}\right|_{\mathbf{q}_{o}, \mathbf{s}_{o}}$ is rank-deficient. Therefore, the rows of $\boldsymbol{\Phi}_{\mathbf{q}} \mathbf{q}_{\mathbf{s}}$ are dependent and there exists a set of constants $\mathbf{n}_{o}=\left[\begin{array}{lll}n_{1} & n_{2} & n_{3}\end{array}\right]^{T}$ that satisfy

$$
\left[\Phi_{\mathbf{q}} \mathbf{q}_{\mathrm{s}}\right]^{T} \mathbf{n}_{o}=\mathbf{0}
$$

where $\mathbf{n}_{o}$ is the vector normal to a singular surface at $\left(\mathbf{q}_{o}, \mathbf{s}_{o}\right)$. In addition, the normal acceleration of the end-effector can be written as:

$$
\ddot{x}_{i}=\sum_{j=1}^{n}\left\{\left[\frac{d}{d t}\left(\frac{\partial \Phi_{i}}{\partial q_{j}}\right)\right]\left(\frac{\partial q_{j}}{\partial \lambda_{j}}\right) \dot{\lambda}_{j}+\left(\frac{\partial \Phi_{i}}{\partial q_{j}}\right)\left[\frac{d}{d t}\left(\frac{\partial q_{j}}{\partial \lambda_{j}}\right)\right] \dot{\lambda}_{j}+\left(\frac{\partial \Phi_{i}}{\partial q_{j}}\right)\left(\frac{\partial q_{j}}{\partial \lambda_{j}}\right) \ddot{\lambda}_{j}\right\}
$$

where

$$
\frac{d}{d t}\left(\partial \Phi_{i} / \partial q_{j}\right) \equiv\left(\partial^{2} \Phi_{i} / \partial q_{\ell} \partial q_{j}\right)\left(\partial q_{\ell} / \partial \lambda_{\ell}\right) \dot{\lambda}_{\ell}
$$

where $\ell=1, \ldots, n$ and $\quad \frac{d}{d t}\left(\partial q_{j} / \partial \lambda_{j}\right) \equiv\left(\partial^{2} q_{j} / \partial \lambda_{\ell} \partial \lambda_{j}\right) \dot{\lambda}_{\ell}$

Expanding terms in the form of joint rates and collecting on similar terms yields

$$
\ddot{x}_{i}=\sum_{j=1}^{n}\left\{\dot{\lambda}_{k}\left[\left(\frac{d q_{k}}{d \lambda_{k}}\right)\left(\frac{\partial^{2} \Phi_{i}}{\partial q_{k} \partial q_{j}}\right)\left(\frac{\partial q_{j}}{\partial \lambda_{k}}\right)+\left(\frac{\partial \Phi_{i}}{\partial q_{j}}\right)\left(\frac{\partial^{2} q_{j}}{\partial \lambda_{k} \partial \lambda_{j}}\right)\right] \dot{\lambda}_{j}+\left(\frac{\partial \Phi_{i}}{\partial q_{j}}\right)\left(\frac{\partial q_{j}}{\partial \lambda_{j}}\right) \ddot{\lambda}_{j}\right\}
$$

where $k=1, \ldots, n$. Written in matrix form, the acceleration is

$$
[\ddot{\mathbf{x}}]_{i}=\dot{\lambda}^{T} \mathbf{q}_{\lambda}{ }^{T}\left[\Phi_{i}\right]_{\mathbf{q q}} \mathbf{q}_{\mathbf{\lambda}} \dot{\lambda}+\dot{\lambda}^{T} \sum_{i=1}^{n}\left(\frac{d \Phi_{i}}{d q_{i}}\left[q_{i}\right]_{\lambda \lambda}\right) \dot{\boldsymbol{\lambda}}+\left[\Phi_{i}\right]_{\mathbf{q}} \mathbf{q}_{\mathbf{\lambda}} \ddot{\boldsymbol{\lambda}}
$$

where $\left[\Phi_{i}\right]_{\mathbf{q q}}=\left[\begin{array}{ccc}\partial \Phi_{i} / \partial q_{1} \partial q_{1} & \ldots & \partial \Phi_{i} / \partial q_{1} \partial q_{n} \\ \cdot & . & \cdot \\ \partial \Phi_{i} / \partial q_{n} \partial q_{1} & \ldots & \partial \Phi_{i} / \partial q_{n} \partial q_{n}\end{array}\right]$ and $\left[q_{i}\right]_{\lambda \lambda}=\left[\begin{array}{ccc}\partial q_{i} / \partial \lambda_{1} \partial \lambda_{1} & \ldots & \partial q_{i} / \partial \lambda_{1} \partial \lambda_{n} \\ \cdot & . & \cdot \\ \partial q_{i} / \partial \lambda_{n} \partial \lambda_{1} & \ldots & \partial q_{i} / \partial \lambda_{n} \partial \lambda_{n}\end{array}\right]$.

To obtain the normal acceleration, $\ddot{\mathbf{x}}$ is projected onto the normal $\mathbf{n}_{o}$. Multiplying both sides of Eq. (25) by the vector $\mathbf{n}_{o}^{T}$ eliminates the last term of the right hand side (definition of the normal as the basis of the null space in Eq. 22).

The component of the normal acceleration is then

where

$$
a_{n}=\mathbf{n}_{o}^{T} \ddot{\mathbf{x}}=\dot{\mathbf{s}}^{T} \mathbf{H}^{*} \dot{\mathbf{s}}
$$

$$
\mathbf{H}^{*}\left(\mathbf{q}_{o}, \mathbf{s}_{o}\right)=\mathbf{q}_{\mathbf{s}}^{T}\left[\mathbf{n}_{o}^{T} \boldsymbol{\Phi}\right]_{\mathbf{q q}} \mathbf{q}_{\mathbf{s}}+\sum_{i=1}^{n} \frac{d\left(\mathbf{n}_{o}^{T} \boldsymbol{\Phi}\right)}{d q_{i}} \cdot\left[q_{i} \rrbracket_{\mathrm{ss}}\right.
$$


In the formulation above (not considering joint limits), definiteness properties of Eq. (17) depended on the $\mathbf{Q}$ matrix. In this extended formulation (considering joint limits), the quadratic form is written in terms of the $\mathbf{Q}^{*}$ matrix as

where

$$
\begin{gathered}
a_{n}-\mathbf{I I}_{\mathbf{p}}^{\prime}=\dot{\mathbf{s}}^{T} \mathbf{Q}^{*} \dot{\mathbf{s}} \\
\mathbf{Q}^{*}=\mathbf{H}^{*}-\mathbf{q}_{\mathbf{s}}^{T} \boldsymbol{\Phi}_{\mathbf{q}}^{T} \mathbf{B}^{T}\left[\mathbf{n}^{T} \boldsymbol{\Psi}\right]_{\mathbf{u u}} \mathbf{B} \boldsymbol{\Phi}_{\mathbf{q}} \mathbf{q}_{\mathbf{s}}
\end{gathered}
$$

Definiteness properties of the quadratic form of Eq. (28) defines the crossability of a singular surface/curve.

\section{Singular Surfaces Due to Active Joint Constraints}

For a singular surface that is due to singularities that are at the upper or lower bounds of an inequality constraint, the surface will definitely be crossable if $\mathbf{Q}^{*}$ has both positive and negative eigenvalues. However, when $\mathbf{Q}^{*}$ is either positive semi-definite or negative semi-definite, the singular surface/curve may still be crossable. On those surfaces, the matrix of the quadratic form loses some terms because joint velocities are zero. Joint velocities at a singular configuration derived from Eq. (19) (i.e., $\dot{q}_{i}=\left[q_{i}^{\max }-q_{i}^{\min } / 2\right] \cos \lambda_{i}$ ) are zero at an upper or lower bound $\left(\lambda_{i}=-\pi / 2\right.$ or $\pi / 2$, i.e., $q_{i}=q_{i}^{\max }$ or $q_{i}=q_{i}^{\min }$ ). Therefore, some terms in $\mathbf{Q}^{*}$ are lost. Supplementary criteria are necessary. To motivate the discussion, consider the two DOF (one prismatic and one revolute) planar manipulator shown in Fig. (1a) with joint limits given by $0 \leq q_{1} \leq 4$ and $(-\pi / 4) \leq q_{2} \leq \pi$.



(a)



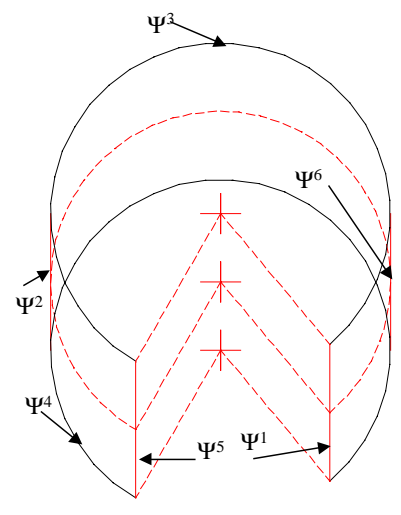

(c)

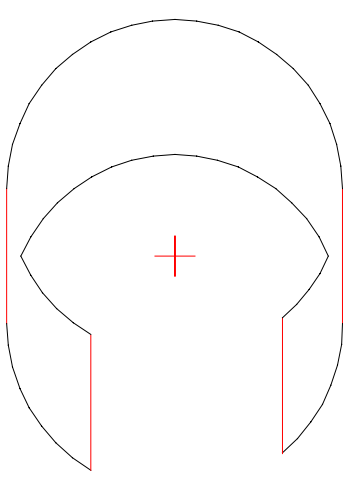

(d)

Figure 1. (a) A two DOF manipulator and (b) Some singular curves due to joint limits (c) All singular curves (d) Workspace envelope

Since $q_{2}$ has its lower and upper bounds as $-\pi / 4$ and $\pi$, the lines $\Psi_{1}$ and $\Psi_{2}$ are the two corresponding singular curves. For the two cases when the end-effector is at points $\mathbf{p}_{1}$ and $\mathbf{p}_{2}$ as shown in Fig. (1b), the joint velocity of $q_{2}$ will always be zero (i.e., $\dot{q}_{2}=(5 \pi / 8) \cos (-\pi / 2)$ ). Therefore, there will not be any component of normal acceleration at point $\mathbf{p}_{1}$ nor at $\mathbf{p}_{2}$. Definiteness properties of $\mathbf{Q}^{*}$ are, therefore, not sufficient to determine all possible directions of motion for these two cases. It should be noted that determination of the workspace is readily carried out first by plotting all singular curves (Fig. 1c) and identifying the boundary (Fig. 1d). 
To address this case, we propose the projection of a variational movement $\delta \mathbf{x}=\mathbf{x}_{q_{i}} \delta q_{i}$ due to $\delta q_{i}$ onto the normal direction $\mathbf{n}$ such that the normal component

$$
\sigma=\mathbf{n}^{T} \mathbf{x}_{q_{i}} \delta q_{i}
$$

determines admissible normal movement, where $\delta q_{i}$ is given a magnitude of \pm 1 as follows

$$
\delta q_{i}= \begin{cases}+1 & \text { if } q_{i} \text { is at lower bound } \\ -1 & \text { if } q_{i} \text { is at upper bound }\end{cases}
$$

Positive values of $\sigma$ in Eq. (30) indicate that the end-effector can admit movement in the positive direction of $\mathbf{n}$.

Thus, for a singular surface that is due to an active joint constraint, Eq. (30) provides supplementary information in lieu of terms lost due to the enforced zero joint velocity. For example, consider the case at point $\mathbf{p}_{1}$ again. Since $\mathbf{Q}^{*}$ at this point has only zero eigenvalues, it is necessary to use Eq. (30) with $\partial q_{2}=+1$ since joint 2 is at the lower limit. Equation (30) yields a positive value, which indicates that the end-effector admits motion into the positive direction of $\mathbf{n}_{1}$.

Another situation arises when the normal vector $\mathbf{n}$ is perpendicular to $\mathbf{x}_{q_{i}}$. In this case, $\sigma$ in Eq. (30) evaluates to zero. Therefore, the direction of normal movement cannot be determined. The normal curvature of the singular surface with respect to the tangent direction of $\mathbf{x}_{q_{i}} \delta q_{i}$ is compared with the normal curvature of the trajectory curve when only $q_{i}$ is varying (other joint variables are held constant).

To determine the normal direction of admissible movement, it is necessary to evaluate the sign of the difference in the normal curvature. Substituting for the first and second fundamental forms (Eqs. 6 and 7) into the normal curvature (Eq. 9) yields

$$
K_{2}-K_{1}=\frac{\delta q_{i}\left[\mathbf{n}^{T} \mathbf{x} \rrbracket_{q_{i} q_{i}} \delta q_{i}\right.}{\delta q_{i} \mathbf{x}_{q_{i}}^{T} \mathbf{x}_{q_{i}} \delta q_{i}}-\frac{\delta \mathbf{u}^{T}\left[\mathbf{n}^{T} \Psi\right]_{\mathbf{u u}} \delta \mathbf{u}}{\delta \mathbf{u}^{T} \Psi_{\mathbf{u}}^{T} \Psi_{\mathbf{u}} \delta \mathbf{u}}
$$

where $\mathrm{K}_{1}$ is the normal curvature of $\Psi_{2}$ with respect to the tangent direction $\mathbf{x}_{q_{i}} \partial q_{i}$, and $\mathrm{K}_{2}$ is the normal curvature of the trajectory curve when $q_{i}$ is varying while other joints are held constant.

The case at point $\mathbf{p}_{2}$ in Fig. (1b), for example, exhibits a zero normal curvature of $\Psi_{2}$. The curvature of the curve due to varying only $q_{2}$ is positive with respect to the normal vector $\mathbf{n}_{2}$. Therefore, the end-effector can move into the side of positive direction of $\mathbf{n}_{2}$ by decreasing $q_{2}$.

Both curves of the right-hand side of Eq. (32) must have the same tangent vector given by Eq. (12) such that

$$
\Psi_{\mathbf{u}} \delta \mathbf{u}=\mathbf{x}_{q_{i}} \delta q_{i}
$$


where $\mathbf{u}$ is the vector of parametric coordinates for the corresponding singular surface/curve. To simplify the calculation, $\delta q_{i}$ can be determined from Eq. (31).

Rearranging Eq. (33) and using the inverse defined in Eq. (18), the vector $\delta \mathbf{u}$ can be written as

$$
\delta \mathbf{u}=\mathbf{B} \mathbf{x}_{q_{i}} \delta q_{i}
$$

Substituting Eq. (34) into Eq. (32) and simplifying yields

$$
\mathrm{K}_{2}-\mathrm{K}_{1}=\frac{\delta q_{i}\left\lceil\mathbf{n}^{T} \mathbf{x} \rrbracket_{q_{i} q_{i}} \delta q_{i}-\delta q_{i} \mathbf{x}_{q_{i}}^{T} \mathbf{B}^{T}\left[\mathbf{n}^{T} \mathbf{x} \rrbracket_{\mathbf{u u}} \mathbf{B} \mathbf{x}_{q_{i}} \delta q_{i}\right.\right.}{\delta q_{i}^{T} \mathbf{x}_{q_{i}}^{T} \mathbf{x}_{q_{i}} \delta q_{i}}
$$

Since the denominator of the right-hand side of Eq. (35) is always positive, the sign is dependent on the numerator. Define the numerator as $\mu$ where

$$
\mu=\delta q_{i}\left[\mathbf{n}^{T} \mathbf{x}\right]_{q_{i} q_{i}} \delta q_{i}-\delta q_{i} \mathbf{x}_{q_{i}}^{T} \mathbf{B}^{T}\left[\mathbf{n}^{T} \mathbf{x}\right]_{\mathbf{u u}} \mathbf{B} \mathbf{x}_{q_{i}} \delta q_{i}
$$

which can be written as

$$
\mu=\left\{\left[\mathbf{n}^{T} \mathbf{x}\right]_{q_{i} q_{i}}-\mathbf{x}_{q_{i}}^{T} \mathbf{B}^{T}\left[\mathbf{n}^{T} \mathbf{x}\right]_{\mathbf{u u}} \mathbf{B} \mathbf{x}_{q_{i}}\right\} \delta q_{i}^{2}
$$

Since $\delta q_{i}^{2}$ is always positive, the sign of $\mathrm{K}_{1}-\mathrm{K}_{2}$ depends on the term inside the brackets. Define

where

$$
\mu=\bar{K} \delta q_{i}^{2}
$$

$$
\bar{K}=\mathbf{x}_{q_{i}}^{T} \mathbf{B}^{T}\left[\mathbf{n}^{T} \mathbf{x}\right]_{\mathbf{u u}} \mathbf{B} \mathbf{x}_{q_{i}}-\left[\mathbf{n}^{T} \mathbf{x}\right]_{q_{i} q_{i}}
$$

Therefore, if $\bar{K}>0$, the end-effector can admit movement into the positive direction of $\mathbf{n}$. If $\bar{K}<0$, the end-effector admits movement into the negative direction of $\mathbf{n}$.

From the above discussion, it can be seen that Eq. (30) and (38) are used to supplement the criteria of the definiteness properties of $\mathbf{Q}^{*}$ to determine crossability of a singular surface with joint limits. Crossability criteria are summarized as follows.

\section{Crossability Criteria}

(1) If $\mathbf{Q}^{*}$ is indefinite (has both positive and negative eigenvalues), the singular surface/curve is crossable for each joint $q_{i}$ which is at its limit.

(2) If $\mathbf{Q}^{*}$ is either positive semi-definite or negative semi-definite, the following additional criteria must be evaluated.

(a) If $\mathbf{n}_{o}$ is not perpendicular to $\mathbf{x}_{q_{i}}$, then $\sigma$ in Eq. (30) must be evaluated

(b) If $\mathbf{n}_{o}$ is perpendicular to $\mathbf{x}_{q_{i}}$, then $\bar{K}$ in Eq. (38) must be evaluated.

(i) If any of $\sigma$ or $\bar{K}$ has a different sign than the nonzero eigenvalues of $\mathbf{Q}^{*}$, the singular surface/curve is crossable.

(ii) If $\sigma$ and $\bar{K}$ have the same sign as the nonzero eigenvalues of $\mathbf{Q}^{*}$, the singular surface/curve is non-crossable.

The direction along which the end-effector can move, either in the positive or negative direction of $\mathbf{n}$, will be in the same sense of signs of $\sigma$ and $\bar{K}$. 


\section{Example 1: A Planar 3-bar Linkage}

This example is presented to demonstrate the implementation of the presented formulation and to validate the results in comparison with those reported by Haug, et al. (1994) for the planar three-bar linkage shown in Fig. 2 with the following imposed limits $-\frac{\pi}{3} \leq q_{i} \leq \frac{\pi}{3} ; i=1 \ldots 3$.

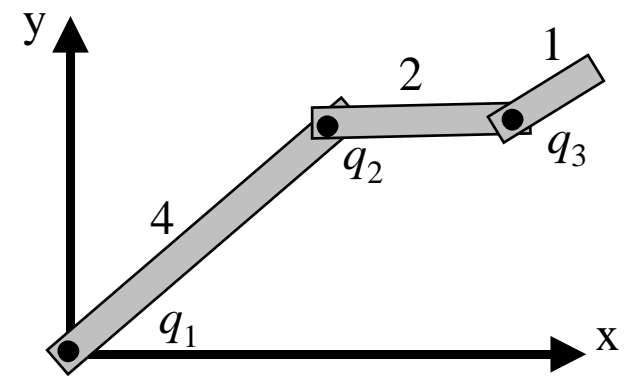

Figure 2. A planar three DOF manipulator

The position vector of a point on the end-effector is defined by

$$
\boldsymbol{\Phi}(\mathbf{q})=\left[\begin{array}{c}
4 \cos q_{1}+2 \cos \left(q_{1}+q_{2}\right)+\cos \left(q_{1}+q_{2}+q_{3}\right) \\
4 \sin q_{1}+2 \sin \left(q_{1}+q_{2}\right)+\sin \left(q_{1}+q_{2}+q_{3}\right)
\end{array}\right]
$$

The $(2 \times 3)$ analytic Jacobian matrix is computed as

$\boldsymbol{\Phi}_{\mathbf{q}}=\left[\begin{array}{lll}-4 \sin q_{1}-2 \sin \left(q_{1}+q_{2}\right)-\sin \left(q_{1}+q_{2}+q_{3}\right) & -2 \sin \left(q_{1}+q_{2}\right)-\sin \left(q_{1}+q_{2}+q_{3}\right) & -\sin \left(q_{1}+q_{2}+q_{3}\right) \\ 4 \cos q_{1}+2 \cos \left(q_{1}+q_{2}\right)+\cos \left(q_{1}+q_{2}+q_{3}\right) & 2 \cos \left(q_{1}+q_{2}\right)+\cos \left(q_{1}+q_{2}+q_{3}\right) & \cos \left(q_{1}+q_{2}+q_{3}\right)\end{array}\right]$

and $\mathbf{q}_{\mathrm{s}}=\left[\begin{array}{ccc}(2 \pi / 6) \cos \lambda_{1} & 0 & 0 \\ 0 & (2 \pi / 6) \cos \lambda_{2} & 0 \\ 0 & 0 & (2 \pi / 6) \cos \lambda_{3}\end{array}\right]$.

Only one singular set results from the $\operatorname{dim}\left[\operatorname{Null}\left(\boldsymbol{\Phi}_{\mathbf{q}}^{T}(\mathbf{q})\right)\right] \geq 1$ defined by $\mathbf{p}_{1}=\left\{q_{2}=0, q_{3}=0\right\}$.

Singularities resulting from the reduced order manipulator (Abdel-Malek and Yeh 1997a) are $S_{2}=\left\{\mathbf{p}_{i}, i=2, \ldots, 7\right\}$ where $\mathbf{p}_{2}=\left\{q_{1}=-\pi / 3, q_{3}=0\right\}, \mathbf{p}_{3}=\left\{q_{1}=\pi / 3, q_{3}=0\right\}$, $\mathbf{p}_{4}=\left\{q_{2}=-\pi / 3, q_{3}=\tan ^{-1}(\sqrt{3} / 2)\right\}, \mathbf{p}_{5}=\left\{q_{2}=\pi / 3, q_{3}=-\tan ^{-1}(\sqrt{3} / 2)\right\}$, $\mathbf{p}_{6}=\left\{q_{3}=-\pi / 3, q_{2}=\tan ^{-1}(\sqrt{3} / 5)\right\}, \mathbf{p}_{7}=\left\{q_{3}=\pi / 3, q_{2}=-\tan ^{-1}(\sqrt{3} / 5)\right\}$. Singularity sets resulting from a combination of joint limits are $\left\{\mathbf{p}_{i}=\left\{q_{i}^{o}, q_{j}^{o}\right\}, i \neq j, i, j=8 \ldots 19\right\}$ where $q_{i}^{o}$ and $q_{j}^{o}$ are the limits of the inequality constraints. Singular curves are shown in Fig. (3a). 


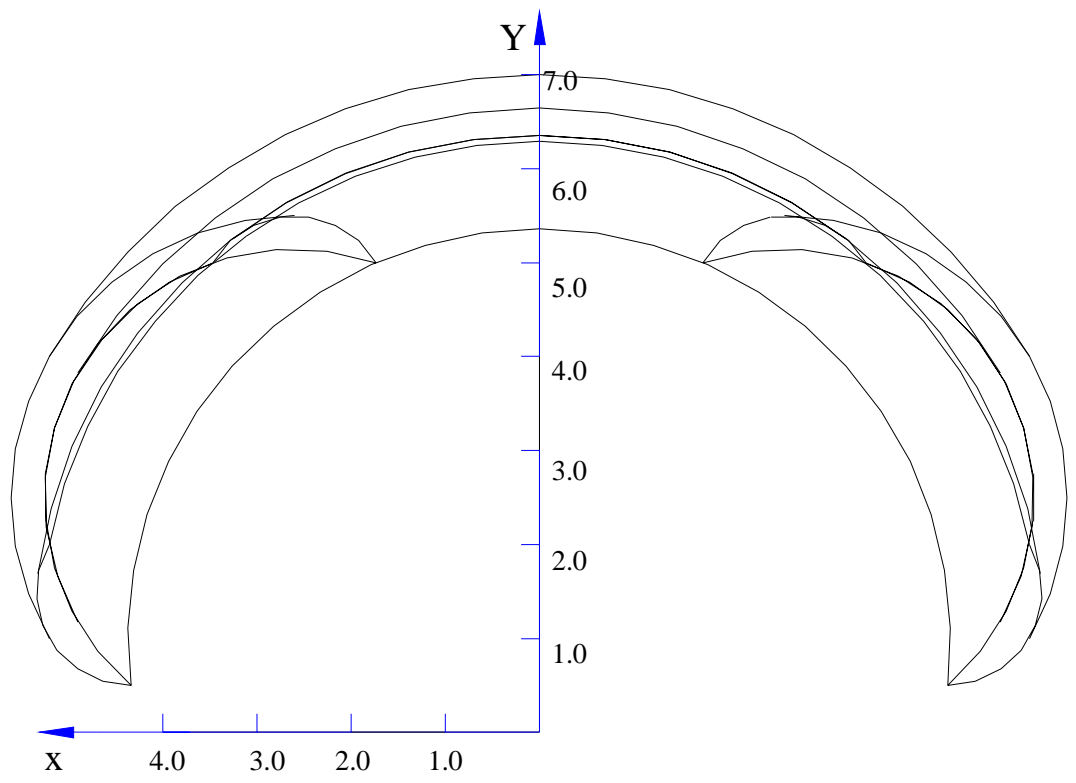

Figure 3. (a) Singular curves of the planar 3-bar linkage

Substituting $\mathbf{p}_{i}$ 's into Eq. (40) yields singular curves $\Psi_{i}$ part of which are shown in Fig. (3b).

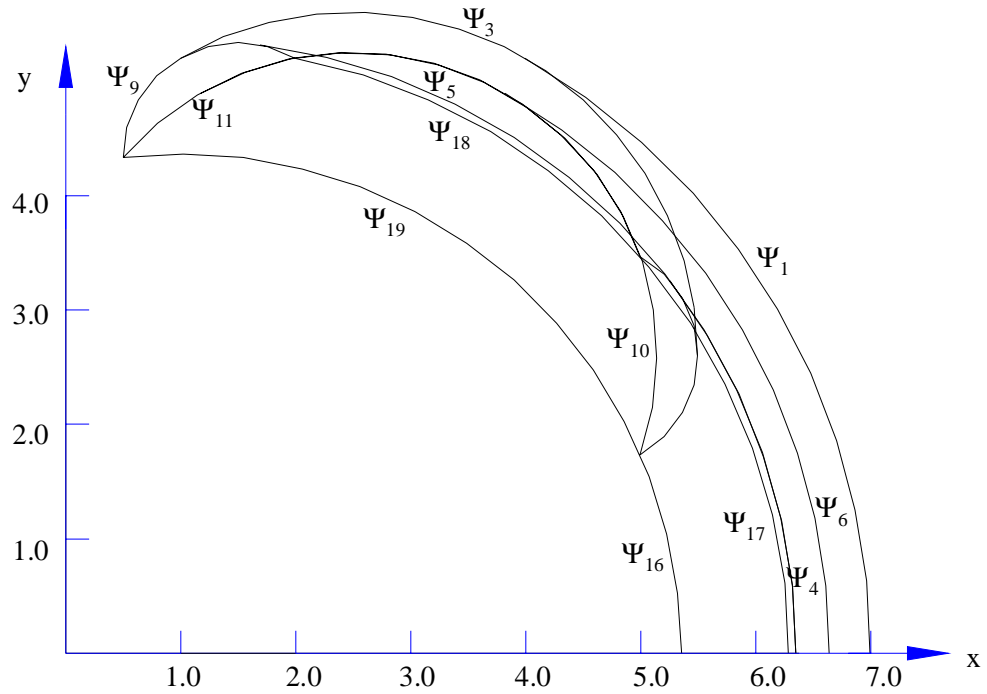

Figure 3. (b) Identifying singular curves

To determine crossable curves, it is necessary to evaluate the criteria above for each independent segment, i.e., curves that cross other curves are segmented. For example, consider a point $\mathbf{p}_{1}$ on curve $\Psi_{19}$ at $q_{1}=\pi / 4, q_{2}=\pi / 3$, and $q_{3}=\pi / 3$. Evaluating the normal $\mathbf{n}$ from the basis of the null space of

$$
\left[\boldsymbol{\Phi}_{\mathbf{q}} \mathbf{q}_{\mathbf{s}}\right]^{T}=\left[\begin{array}{ccc}
-3.4765 & 0 & 0 \\
0.9315 & 0 & 0
\end{array}\right]
$$

at $\mathbf{p}_{1}$ yields the normal $\mathbf{n}=\left[\begin{array}{ll}0.2588 & 0.9659\end{array}\right]^{T}$. Evaluating the matrix $\mathbf{H}^{*}$ of Eq. (27) yields

$$
\mathbf{H}^{*}=\left[\begin{array}{ccc}
-2.4930 & 0 & 0 \\
0 & 2.0944 & 0 \\
0 & 0 & 1.0472
\end{array}\right]
$$


Since this is a planar example, the singular curve is parameterized by only one joint variable (i.e., $\Psi_{19}\left(q_{1}\right)$, therefore, $\mathbf{x}_{q_{1}}$ is a $(2 \times 1)$ matrix. The matrix $\mathbf{E}$ is therefore a $\mathbf{E}=\left[\begin{array}{ll}1 & 0\end{array}\right]$ vector and $\mathbf{B}=\left[\mathbf{E} \Psi_{\mathbf{u}}\right]^{-1} \mathbf{E}=\left[\begin{array}{ll}-0.1992 & 0\end{array}\right]$. The matrix $\mathbf{Q}^{*}$ of the quadratic form is evaluated as

$$
\mathbf{Q}^{*}=\left[\begin{array}{ccc}
0 & 0 & 0 \\
0 & 2.094 & 0 \\
0 & 0 & 1.047
\end{array}\right]
$$

The eigenvalues of $\mathbf{Q}^{*}$ are evaluated $\{0,1.047$, and 2.094$\}$, which indicates a positive semidefinite quadratic form. Since the constraints of joints 2 and 3 are active (upper bounds) and $\mathbf{x}_{q_{2}}$ and $\mathbf{x}_{q_{3}}$ are not perpendicular to $\mathbf{n}$, the additional value of $\sigma$ is evaluated. For $q_{2}$ at its upper limit, $\delta q_{2}=-1$, and $\sigma=\mathbf{n}^{T} \mathbf{x}_{q_{2}} \delta q_{2}=2$ which is positive. For $q_{3}$, choose $\delta q_{3}=-1$ and evaluate $\sigma=\mathbf{n}^{T} \mathbf{x}_{q_{3}} \delta q_{3}=1$ which is also positive. Both values for $\sigma$ are positive (same signs as the eigenvalues of $\mathbf{Q}^{*}$ ). Therefore, surface $\Psi_{19}$ only admits movement into the positive direction of the vector normal $\mathbf{n}$ (i.e., only towards the internal of the workspace) as expected.

To further demonstrate, consider point $\mathbf{p}_{2}$ on $\Psi_{6}$ at $q_{1}=\pi / 8, \quad q_{2}=\tan ^{-1}(\sqrt{3} / 5)$, and $q_{3}=-\pi / 3$. The matrix $\mathbf{Q}^{*}$ of the quadratic form is indefinite (both negative and positive eigenvalues) indicating a crossable curve at $\mathbf{p}_{2}$. Similar analysis is performed for all curve segments. For example, the curve $\Psi_{8}$ is crossable for the segment of $0.7137 \leq q_{3} \leq \pi / 3$; noncrossable for the segment $0 \leq q_{3} \leq 0.7137$; and crossable for $-\pi / 3 \leq q_{3} \leq 0$. The curve $\Psi_{9}$ is non-crossable for the segment of $0 \leq q_{3} \leq \pi / 3$; crossable for the segment $-0.7137 \leq q_{3} \leq 0$; and non-crossable for $-\pi / 3 \leq q_{3} \leq-0.7137$. Crossable curves are shown dotted in Fig. (4a) while non-crossable lines are shown solid. Directions of admissible motion are shown by arrows on each curve segment in Fig. (4b).


Figure 4. (a) Singular curves (b) Admissible normal movement directions 


\section{Example 2: A Four DOF Manipulator}

The manipulator shown in Fig. 5 comprises two revolute and two prismatic joints. Joint limits are imposed as $0 \leq q_{1} \leq 2 \pi, 20 \leq q_{2} \leq 50,(-\pi / 4) \leq q_{3} \leq \pi$, and $10 \leq q_{4} \leq 20$.



Figure 5. A four DOF manipulator with prismatic joints

For a point on the end-effector, the position vector is

$$
\boldsymbol{\Phi}(\mathbf{q})=\left[\begin{array}{llll}
q_{4} \cos q_{1} \cos q_{3}+30 \cos q_{1} & q_{4} \sin q_{1} \cos q_{3}+30 \sin q_{1} & q_{4} \sin q_{3}+q_{2}
\end{array}\right]^{T}
$$

Inequality constraints are parameterized as $q_{1}=\pi+\pi \sin \lambda_{1}, \quad q_{2}=35+15 \sin \lambda_{2}$, $q_{3}=\frac{3 \pi}{8}+\frac{5 \pi}{8} \sin \lambda_{3}$, and $q_{4}=15+5 \sin \lambda_{4}$. The Jacobian is derived as

$$
\boldsymbol{\Phi}_{\mathbf{q}}=\left[\begin{array}{cccc}
-q_{4} \sin q_{1} \cos q_{3}-30 \sin q_{1} & 0 & -q_{4} \cos q_{1} \sin q_{3} & \cos q_{1} \cos q_{3} \\
q_{4} \cos q_{1} \cos q_{3}+30 \cos q_{1} & 0 & q_{4} \sin q_{1} \sin q_{3} & \sin q_{1} \cos q_{3} \\
0 & 1 & q_{4} \cos q_{3} & \sin q_{3}
\end{array}\right]
$$

The rank-deficiency criteria of the Jacobian yields no singular sets since singularities obtained from the determinants of the sub-Jacobians do not satisfy the inequality constraints. The reduced-order manipulator set (substituting a joint limit value) yields four singular sets as $\mathbf{p}_{1}=\left\{q_{3}=0, q_{4}=10\right\}, \mathbf{p}_{2}=\left\{q_{3}=\pi, q_{4}=10\right\}, \mathbf{p}_{3}=\left\{q_{3}=0, q_{4}=20\right\}, \mathbf{p}_{4}=\left\{q_{3}=\pi, q_{4}=20\right\}$.

Other singular sets result from active joint constraints: $\mathbf{p}_{5}=\left\{q_{2}=20, q_{3}=-\pi / 4\right\}$, $\mathbf{p}_{6}=\left\{q_{2}=20, q_{3}=\pi\right\}, \quad \mathbf{p}_{7}=\left\{q_{2}=20, q_{4}=10\right\}, \quad \mathbf{p}_{8}=\left\{q_{2}=20, q_{4}=20\right\} \quad \mathbf{p}_{9}=\left\{q_{2}=50, q_{3}=-\pi / 4\right\}$, $\mathbf{p}_{10}=\left\{q_{2}=50, q_{3}=\pi\right\}, \quad \mathbf{p}_{11}=\left\{q_{2}=50, q_{4}=10\right\}, \quad \mathbf{p}_{12}=\left\{q_{2}=50, q_{4}=20\right\}, \quad \mathbf{p}_{13}=\left\{q_{3}=-\pi / 4, q_{4}=10\right\}, \quad$ and $\mathbf{p}_{14}=\left\{q_{3}=-\pi / 4, q_{4}=20\right\}$. Substituting each singularity set into Eq. (42) yields parametric equations of singular surfaces in $\mathbf{R}^{3}$. Figure 6 is a cross-section of the workspace volume depicting all singular surfaces. 


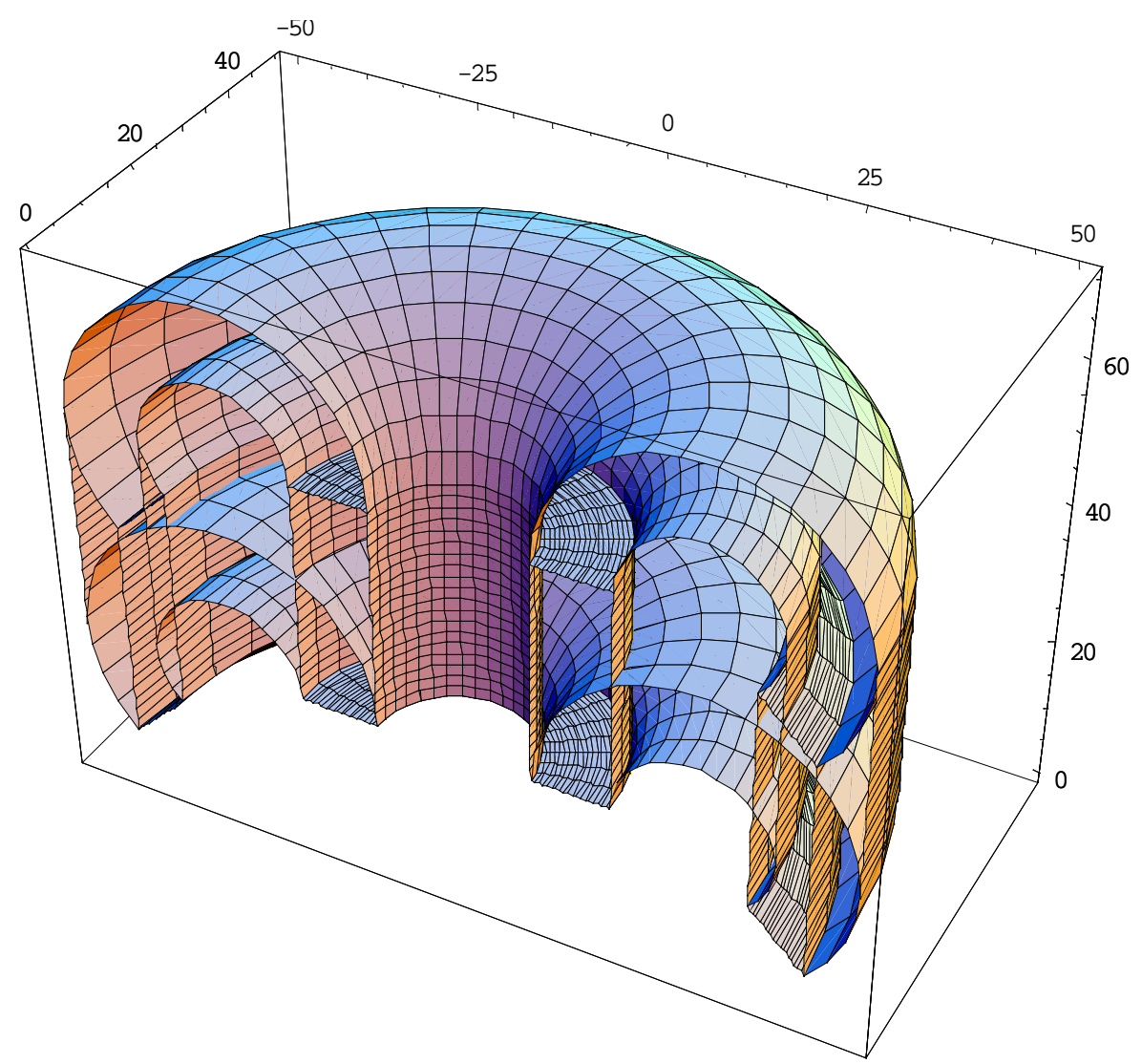

Figure 6. A cross-section of the workspace depicting all singular surfaces

Figure 7a depicts a cross section (a longitudinal slice) due to $q_{1}=\pi / 2$. To study the crossability of singular surfaces, consider surface $\Psi_{11}$. Let $\mathbf{p}_{1}$ be a point on $\Psi_{11}$ at $q_{1}=\pi / 2$, $q_{2}=50, q_{3}=3 \pi / 4$, and $q_{4}=10$. The vector normal at $\mathbf{p}_{1}$ is calculated using Eq. (22) as $\mathbf{n}=\left[\begin{array}{lll}0 & -\sqrt{2} / 2 & -\sqrt{2} / 2\end{array}\right]^{T}$. The Eigenvalues of $\mathbf{Q}^{*}$ are $\{0,0,-10.6,5.0\}$. Since the quadratic form is indefinite, the singular surface is crossable at this point.

For another point $\mathbf{p}_{2}$ on surface $\Psi_{11}$ at $q_{1}=\pi / 2, q_{2}=50, q_{3}=-\pi / 6$, and $q_{4}=10$. The vector normal is $\mathbf{n}=\left[\begin{array}{lll}0 & -\sqrt{3} / 2 & 1 / 2\end{array}\right]^{T}$. Eigenvalues of $\mathbf{Q}^{*}$ are $\left\{\begin{array}{ll}0,0,-7.5,-5.0\end{array}\right\}$. Since the quadratic form is negative semidefinite, the additional criteria should be evaluated. For this surface, the joint variables $q_{2}$ and $q_{4}$ are at their limits. The additional criteria are also negative indicating a non-crossable surface at this point. The end-effector can move only in the direction of the negative normal vector. In fact, the region of surface $\Psi_{11}$ for which $-\pi / 4 \leq q_{3} \leq 0$, is non-crossable. The region for which $0 \leq q_{3} \leq \pi$ is crossable. Similarly, for surface $\Psi_{12}$, the region for which $-\pi / 4 \leq q_{3} \leq 0$ is crossable and for which $0 \leq q_{3} \leq \pi$ is non-crossable. Figure $7 \mathrm{~b}$ depicts crossable (solid) and noncrossable (dotted) traces of surfaces of a cross-section of the workspace. Arrows indicating admissible normal movements are shown in Fig. (7c). An arrow pointing in one direction indicates the admissible motion of the end-effector in that direction. 

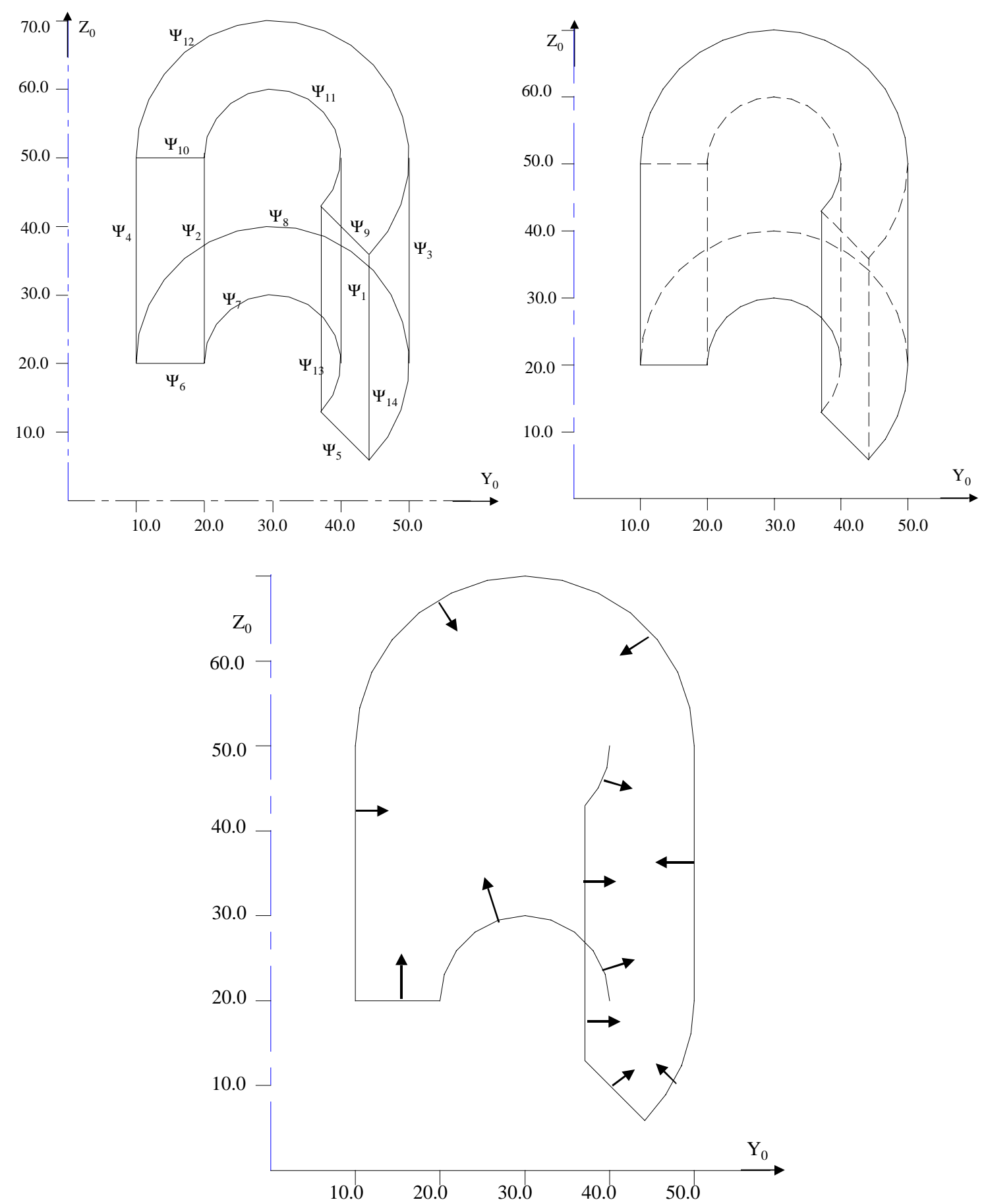

Figure 7. (a) A cross-section of the workspace at $q_{1}=\pi / 2$, (b) crossable and noncrossable surfaces, and (c) Admissible directions of normal movement

\section{Conclusions}

The formulation presented in this paper and illustrated using planar and spatial manipulators with joint limits, demonstrates a complete solution to the problem of determining singular behavior in the workspace of open-chain mechanisms. New results regarding the admissibility of movement of the end-effector across singular surfaces are presented. Validation of these 
It was shown that the crossability of a singular surface can be determined from the definiteness properties of a quadratic form based on acceleration analysis. It was also shown that singular surfaces that are due to active joint constraints require additional criteria to determine their crossability. The additional criterion is needed because of the loss of information in the quadratic term due to zero enforced joint velocity on the singular surface. Two supplementary criteria were derived based on the direction of the normal curvature for surfaces that have active constraints. Two conditions were shown to exist. The first is based upon the projection of a differential motion on the normal to the singular surface. The second method is based upon comparing the normal curvatures of the singular surface with that of a trajectory due to the variation of the joint that reached the limit.

The planar and spatial examples introduced represent the first complete treatment of crossability analysis of singular surfaces in an analytical formulation. Although control problems and manipulability measures are a natural extension of this work, they have not been addressed in this paper, but are the subject of current studies.

\section{Acknowledgments}

The authors gratefully acknowledge the support of the Department of Defense under grant number DAAE07-94-C-R094 and the Old Gold Award from the University of Iowa.

\section{References}

Abdel-Malek, K and Yeh, HJ, 1997a, "Analytical Boundary of the Workspace for General Three Degree-of-Freedom Mechanisms," International Journal of Robotics Research. Vol. 16, No. 2, pp. 198-213.

Abdel-Malek, K and Yeh, HJ, 1997b, "Geometric Representation of the Swept Volume Using Jacobian Rank-Deficiency Conditions," Computer-Aided Design, Vol. 29, No. 6, pp. 457468.

Abdel-Malek, K, Adkins, F, Yeh, HJ, and Haug, EJ, 1997, "On the Determination of Boundaries to Manipulator Workspaces," Robotics and Computer-Integrated Manufacturing, Vol. 13, No. 1, pp.63-72.

Abdel-Malek, K, and Yeh, HJ, 1997c, "Path Trajectory Verification for Robot Manipulators in a Manufacturing Environment" IMechE Journal of Engineering Manufacture, Vol. 211, part B, pp. 547-556.

Abdel-Malek, K, Yeh, HJ, and Khairallah, N, 1999 "Workspace, Void, and Volume Determination of the General 5DOF Manipulator, Mechanics of Structures and Machines, Vol. 27, No. 1, pp. 91-117.

Ahmad, S. and Luo, S. 1988. Analysis of kinematic singularities for robot manipulators in Cartesian coordinate parameters. Proceedings of the IEEE Int. Conf. on Rob. and Autom., Philadelphia, PA, 840-845.

Allgower, E. L. and Georg, K. 1990. Numerical Continuation Methods: An Introduction. Springer-Verlag, Berlin.

Bulca, F.; Angeles, J.; Zsombor-Murray, P.J., 1999, "On the workspace determination of spherical serial and platform mechanisms", Mechanism \& Machine Theory, v34 n3, pp. 497-512. 
Burdick, J. W. 1995. Recursice method for finding revolute-jointed manipulator singularities. ASME J. Mech. Des., 117:55-63.

Burdick, J.W., 1991, "A Classification of 3r regional manipulator singularities and geometries", Proceedings of the IEEE International Conference on Robotics and Automation, Sacramento, CA, pp. 2670-2675.

Burdick, J.W., 1992, "A recursive method for finding revolute-jointed manipulator singularities", Proceedings of the IEEE International Conference on Robotics and Automation, Nice, France, pp. 448-453.

Chevallereau, C. and Daya, B. 1994, "A new method for robot control in singular configurations with motion in any Cartesian direction", Proceedings of the IEEE International Conference on Robotics and Automation, Vol. 4, San Diego, CA, pp. 2692-2697.

Chevallereau, C., 1996, "Feasible trajectories for a non redundant robot at a singularity" Proc. of IEEE International Conference on Robotics and Automation, Minneapolis, MN, pp. 1871-1876.

Cugini, U. Radi, S. and Rizzi, C. 1994. A system for parametric surface intersection. in Computer-Aided Surface Geometry and Design, The Mathematics of Surfaces (A. Bowyer), Oxford University Press.

Denavit, J. and Hartenberg, R.S., 1955. A kinematic notation for lower-pair mechanisms based on matrices. Journal of Applied Mechanics, 77:215-221.

Farin, G. 1993. Curves and Surfaces for Computer-Aided Geometric Design, Academic Press, Inc, San Diego, CA.

Fu, K. S. Gonzalez, R. C. and Lee, C. S. G. 1987. Robotics: Control, Sensing, Vision, and Intelligence, McGraw-Hill, Inc., New York.

Gorla, B. 1981. Influence of the control on the structure of a manipulator from a kinematic point of view. Proc. 4th Symp. Thoery and Practice of Rob. Manipulators, Zaborow, Poland, 30-46.

Haug, E. J. Adkins, F. A. Qiu, C. C. and Yen, J. 1995. Analysis of barriers to control of manipulators within accessible output sets. Proceedings of the 20th ASME Design Engineering Technical Conference, Boston, MA, 82:697-704.

Haug, E. J. Luh, C. M. Adkins, F. A. and Wang, J. Y. 1994. Numerical algorithms for mapping boundaries of manipulator workspaces. Advances in Design Automation, ASME DE 69(2):447-459.

Karger, A. 1995. Classification of robot-manipulators with only singular configurations. Mechanism and Machine Theory, 30: 727-736.

Kumar, A. and Waldron, K. J. 1981. The workspaces of a mechanical manipulator. ASME J. of Mech. Des., 103(3):665-672.

Lai, Z. C. and Yang, D. C. H. 1984. On the singularity analysis of simple six-link manipulators ASME Paper No. 84-DET-220.

Lai, Z. C. and Yang, D. C. H. 1986. A new method for the singularity analysis of simple six-link manipulators. Int. J. of Rob. Res., 5(2):66-74.

Lipkin, H. and Pohl, E. 1991. Enumeration of singular configurations for robotic manipulators. ASME J. Mech., Trans., and Autom. in Des., 113:272-279.

Litvin, F. L. Fanghella, P. Tan, J. and Zhang, Y. 1986. Singularities in motion and dispplacement functions of spppatial linkages. ASME J. of Mech., Trans., and Autom. in Des., 108(4):516-523. 
Litvin, F. L. Yi, Z. Castelli, V. P. and Innocenti, C. 1986. Singularities, configurations, and displacement functions for manipulators. International Journal of Robotics Research, 5(2):52-65.

Merlet, J. P. 1989. Singular configurations of parallel manipulators and Grassman geometry. Int. J. of Rob. Res., 8:45-56.

Nakamura, Y., 1991, Advanced Robotics: Redundancy and Optimization, Addison-Wesley Publishing Company.

Nielsen, L. deWitt, C. C. and Hagander, P. 1991. Controllability issues of robots in singular configurations. Proceedings of IEEE Int. Conf. on Rob. and Autom., Sacramento, CA.

Oblak, D. and Kohli, D., 1988. Boundary surfaces, limit surfaces, crossable and noncrossable surfaces in workspace of mechanical manipulators. ASME Journal of Mechanisms, Transmissions, and Automation in Design, (110):389-396.

Pai, D. K. and Leu, M. C. 1992, "Generecity and singularities of robot manipulators. IEEE Transactions on Robotics and Automation, Vol. RA-8, pp. 545-559.

Pai, D. K. and Leu, M. C. 1992. Generecity and singularities of robot manipulators. IEEE Trans. on Rob. Autom., 8:545-559.

Sciavicco, L. and Siciliano, B. 1996. Modeling and Control of Robot Manipulators. McGrawHill, Inc., New York.

Sefrioui, J. and Gosselin, C. M. 1994. Determination of the singularity loci of spherical threedegree-of-freedom parallel manipulators. Mechanism and Machine Theory, 30:559-579.

Sefrioui, J. and Gosselin, C. M. 1995. On the quadratic nature of the singularity curves of planar three-degree-of-freedom parallel manipulators. Mechanism and Machine Theory, 30:533-551.

Shamir, T. 1990. The singularities of redundant robot arms. International Journal of Robotics Research, 2(1):113-121.

Shu, M. Kohli, D. and Dwivedi, S. H. 1986. Proceedings of the 6th World Congress on Theory of Machines and Mechanisms, New Delhi, India, 988-993.

Soylu, R. and Duffy, J. 1988. Hypersurfaces of special configurations of serial manipulators and related concepts. Part II: Passive joints, configurations, component manifolds and some applications. Journal of Robotic Systems, 5:31-53.

Spanos, J. and Kohli, D., 1985, "Workspace Analysis of Regional Sttructure of Manipulators," ASME J. of Mech. Trans. and Aut. in Design, Vol. 107, pp. 219-225.

Sugimoto, K. Duffy, J. andHunt, K. H. 1982. Special configurations of spatial mechanisms and robot arms. Mechanism and Machine Theory, 117(2):119-132.

Tourassis, V. D. and Ang, M. H. 1992. Identification and analysis of robot manipulator singularities. Int. J. of Rob. Res., 11:248-259.

Waldron, K. J. 1987. Operating barriers within the workspace of manipulators. Proceedings of the Society of Manufacturing Engineers, Chicago, IL, Robots II/17th ISIR, 8:35-46.

Waldron, K. J. Wang, S. L. and Bolin, S. L. 1985. A study of the Jacobian matrix of serial manipulators. ASME J. of Mech., Trans., and Autom. in Des., 107(2):230-238.

Wang, S. L. and Waldron, K. J. 1987. Study of the singular configurations of serial manipulators. ASME J. of Mech., Trans., and Autom. in Des., 109(1):14-20. 